\title{
A NON-RESONANT RF CAVITY LOADED WITH AMORPHOUS ALLOY FOR PROTON CANCER THERAPY
}

\author{
Y. Makita ${ }^{\#}$, H. Harada, T. Nagayama, H. Tsuchidate, C. Tsukishima, and K. Yoshida \\ Mitsubishi Electric Corporation \\ 8-1-1 Tsukaguchi-Honmachi, Amagasaki, Hyogo 661-8661, Japan
}

\begin{abstract}
A non-resonant rf cavity loaded with amorphous alloy cores has been designed and tested. The cavity has a reentrant structure loaded with 8 amorphous alloy toroidal core and its characteristic impedance is designed as 450 $\Omega$. The rf power is fed by $1 \mathrm{~kW}$ solid state amplifier using a step-up transformer with 1:9 impedance ratio. In the high power test, an accelerating gap voltage of more than $900 \mathrm{~V}$ was measured with input power of $1 \mathrm{~kW}$ in the frequency range of 1 to $10 \mathrm{MHz}$. The voltage standing wave ratio (VSWR) was less than 2.0. The results prove that the cavity may be used successfully within a compact proton synchrotron for a cancer therapy facility.
\end{abstract}

\section{INTRODUCTION}

Cancer is the leading cause of death. In fact, more than one in four die of cancer at present in Japan. Use of the proton beam in cancer therapy is a promising mode of treatment because of its ability to selectively destroy cancer cells while causing minimum damage to the surrounding healthy tissues. At present, research in proton and ion beam cancer treatment is being pursued at several institutions in Japan.

At Mitsubishi Electric, we have been developing a cancer treatment facility based on the proton synchrotron. It is required that such a system be easily operated without the assistance of accelerator experts. Low cost and compactness are also important features for widespread use in the hospital environment.

Because of such considerations, a non-resonant cavity [1] is the natural choice for the accelerating system since the need for a tuning element is eliminated, leading to a substantial reduction in the system complexity. The performance of such a cavity is determined to a large degree by the choice of the core which loads the gap. We chose an amorphous material having a large magnetic permeability, high saturation flux density and a high Curie temperature in order to attain a high gap voltage and stability against the heat load.

We have constructed such a cavity and performed highpower tests to measure its properties such as the impedance, accelerating voltage and temperature rise during operation. In this paper, we discuss the design features of this cavity along with the results of these measurements.

"Email: yo@wel.crl.melco.co.jp

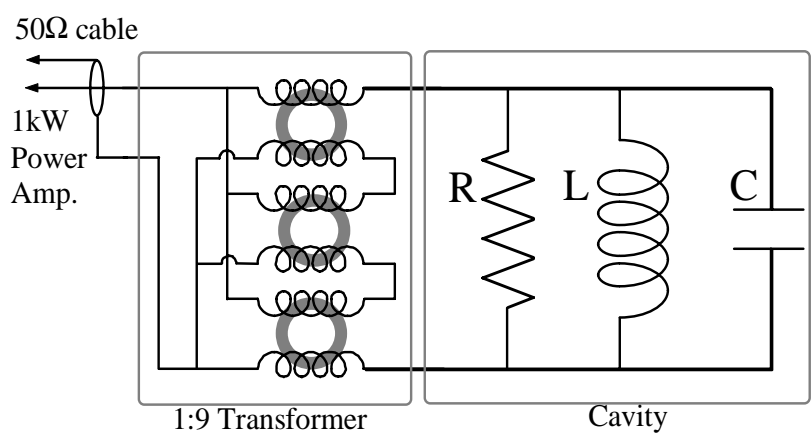

Figure 1 The circuit diagram of the RF system

\section{RF CAVITY}

Figure 1 is a circuit diagram of the RF system including the RF cavity, the power amplifier and the transformer. The cavity is described as a parallel resonant circuit with impedance $Z_{\text {cav }}$ given by the following equations;

$$
\frac{1}{Z_{c a v}}=\frac{1}{R}+\frac{1}{j \omega L}+j \omega C
$$

where,

$$
R=\frac{|\mu|^{2}}{\mu^{\prime}} \omega L_{0}, \quad L=\frac{|\mu|^{2}}{\mu^{\prime \prime}} L_{0}, \quad C=C_{g}
$$

$\mu=\mu^{\prime}-j \mu^{\prime \prime}$

$\mu^{\prime}=$ the real part of the complex permeability

$\mu^{\prime \prime}=$ the imaginary part of the complex permeability

$L_{0}=$ the inductance of the cavity with no core

$C_{g}=$ the capacitance of the cavity

To design a non-resonant cavity, it is desirable to keep the absolute value of the complex impedance flat in the operating frequency range. This is accomplished by using the magnetic property of the core material above the Snoek's limit. i.e. in this region both the real and imaginary parts of the magnetic permeability falls inversely with frequency, hence the first two terms in equation (1) becomes independent of frequency. Furthermore, a high value of permeability is also preferred from the point of view of power economy.

In the past, the ferrite core has been commonly chosen as the loading material [2]. However, by selecting an amorphous material it is possible to obtain a higher permeability and at the same time a higher saturation flux density and a higher Curie temperature. Thus the amorphous core is suited in fabricating a more compact cavity. 


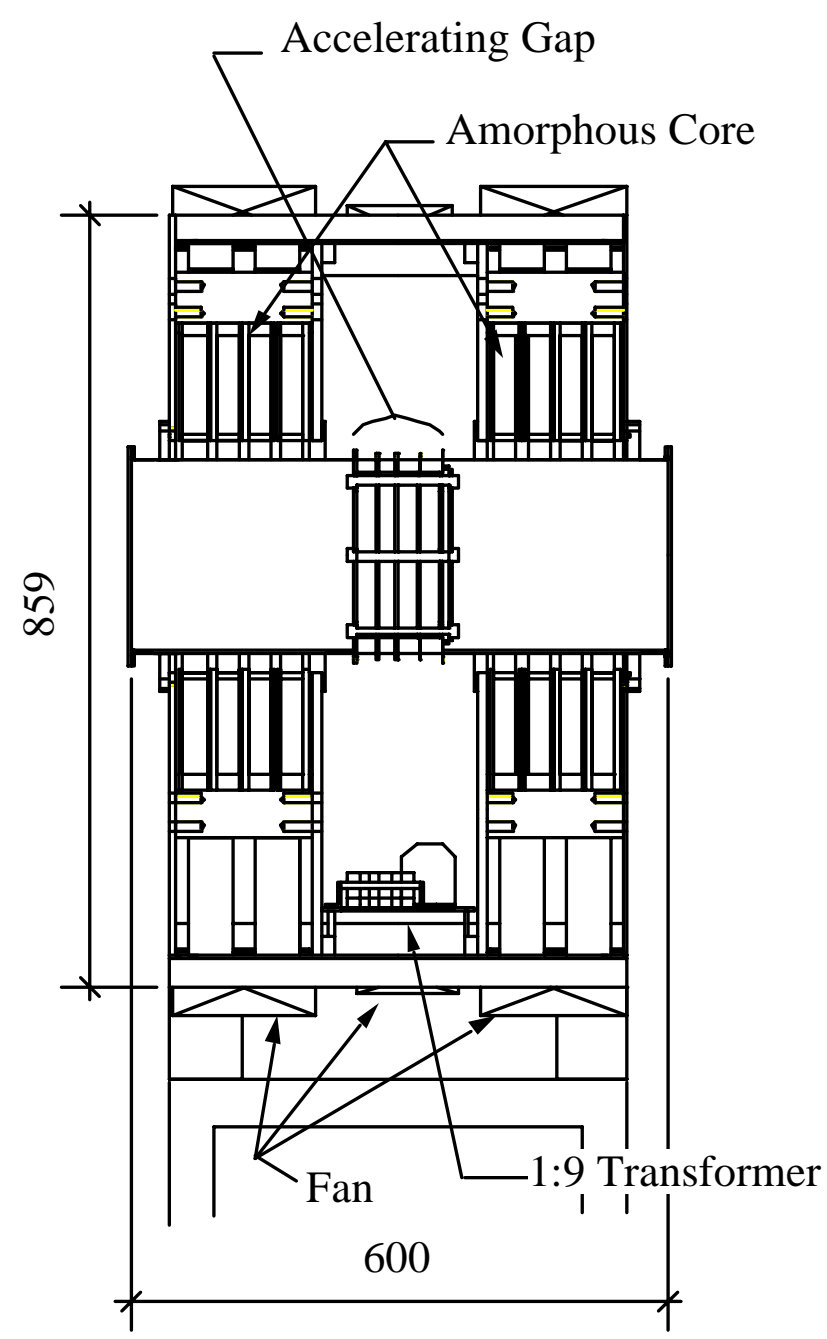

Figure 2 The schematic diagram of the RF cavity

The schematic diagram of the cavity is shown in Figure 2. The cavity has a single accelerating gap in the middle, consisting of two quarter-wavelength coaxial resonators each loaded with four amorphous cores. The design parameters of the cavity are summarized in Table 1.

Since the amplifier has a driving impedance of $50 \Omega$, a step-up transformer with a 1:9 impedance ratio was used to match to the cavity impedance [3].
Table 1 Design specifications of the cavity

\begin{tabular}{|l|c|}
\hline operation frequency & $1-10 \mathrm{MHz}$ \\
\hline cavity impedance & $450 \Omega$ \\
\hline accelerating voltage & $900 \mathrm{~V}$ \\
\hline disipated power & $1 \mathrm{~kW}$ \\
\hline cavity dimension & $\mathrm{H}: 830$ \\
\cline { 2 - 2 }$[\mathrm{mm}]$ & $\mathrm{L}: 600$ \\
\hline & $\mathrm{W}: 700$ \\
\hline duct dimension & $\phi: 216$ \\
\hline [mm] & $\mathrm{L}: 600$ \\
\hline amorphous core & $\phi$ in: 270 \\
\hline [mm] & $\phi$ out:487 \\
\cline { 2 - 2 } & $\mathrm{t}: 25.5$ \\
\hline number of cores & 8 \\
\hline
\end{tabular}

\section{MEASUREMENTS}

The fabricated cavity was not intended for testing under vacuum. Hence MC Nylon was used as the accelerating gap, rather than ceramic. The gap length was made variable but kept at $92 \mathrm{~mm}$ for all measurements described here. The total cavity length was required to be $600 \mathrm{~mm}$ or less by the circumference of the synchrotron.

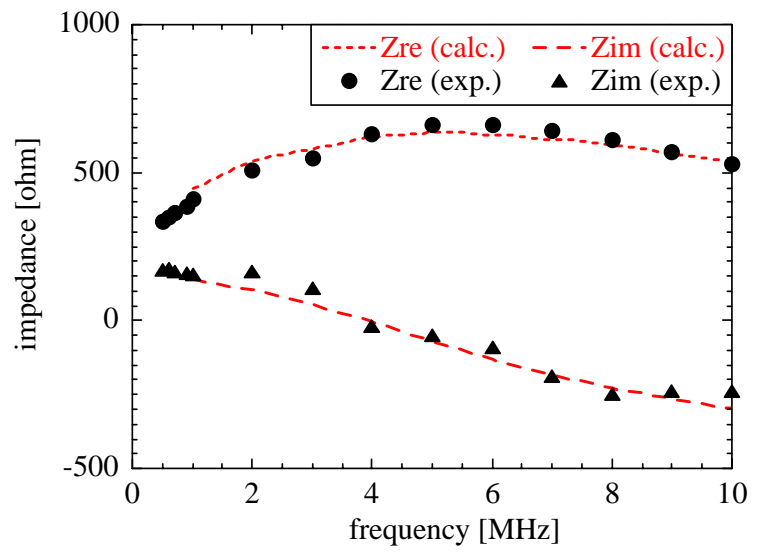

Figure 3 The complex impedance of the cavity

The complex impedance of the cavity was measured with a network analyzer. The result is given in Figure 3. The data are compared to a calculation using lumped circuit approximation. A good agreement is obtained between measured and calculated results in all cases.

To confirm that the transformer works properly, the impedance was measured with a $450 \Omega$ resistor load connected to the transformer. The results are shown in Figure 4.

A high-power test was performed using a $1 \mathrm{~kW}$ solidstate amplifier. For the measurement, high voltage probes were directly attached to both sides of the gap. An accelerating gap voltage of more than $900 \mathrm{~V}$ was obtained in the frequency range of 1 to $10 \mathrm{MHz}$ as shown in Figure 5. A good agreement is obtained between measured and calculated results. 


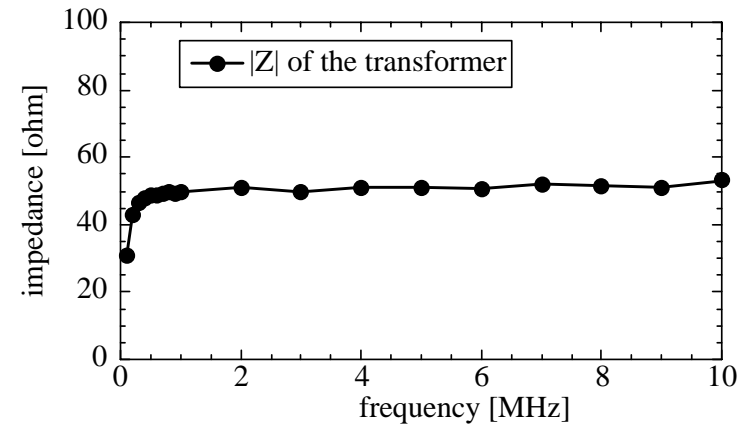

Figure 4 The impedance of the transformer

VSWR was measured with a power meter during the high-power test. The frequency dependence of the VSWR is given in Figure 5. The measured VSWR was lower than 1.9 throughout the frequency range, which corresponds to less than $10 \%$ reflected power from the cavity.

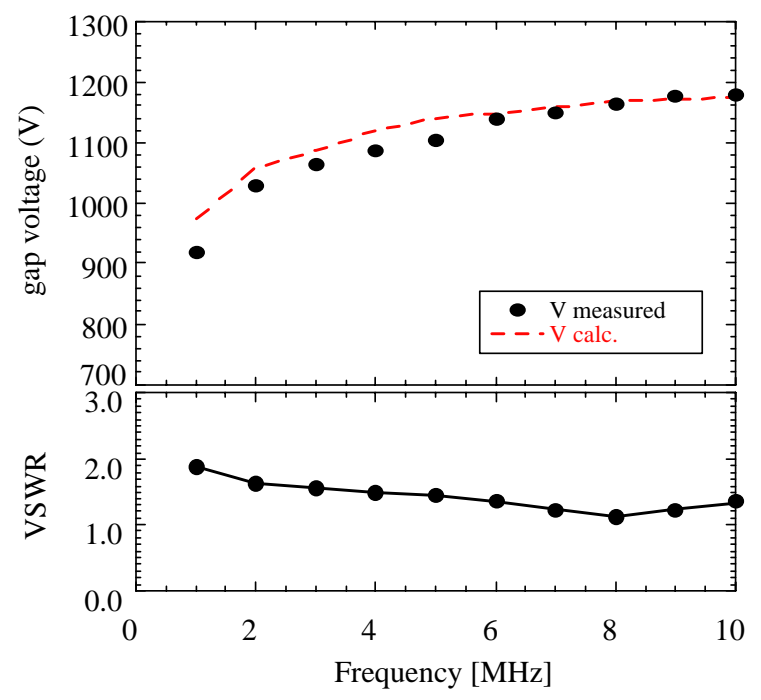

Figure 5 The accelerating voltage and VSWR of the cavity

A heat-up test was also performed. The temperature was monitored with thermocouples attached to the surface of the amorphous core of the cavity and to the ferrite core of the impedance matching transformer during a highpower operation which lasted for one hour. The measured data are shown in Figure 6. Due to forced air cooling with 8 fans for the amorphous cores and 4 small fans for the ferrite cores, the temperature increases were as small as $14 \operatorname{deg} \mathrm{C}$ and $10 \operatorname{deg} \mathrm{C}$ respectively.

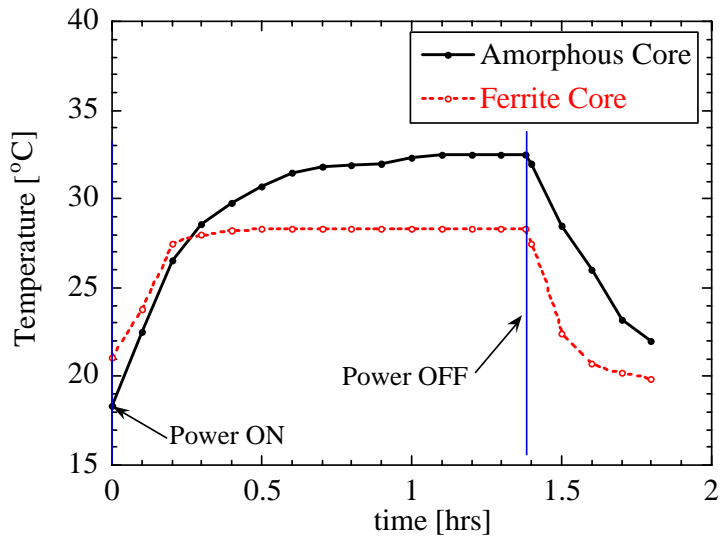

Figure 6 The temperature of amorphous core of the cavity and ferrite core of the transformer

\section{CONCLUSION}

A non-resonant cavity loaded with amorphous cores has been fabricated and tested. An accelerating gap voltage of more than $900 \mathrm{~V}$ was measured with a $1 \mathrm{~kW}$ amplifier in the frequency range of 1 to $10 \mathrm{MHz}$. The reflected power from the cavity was confirmed to be less than $10 \%$ for a measured VSWR of less than 1.9. The temperature rise was observed to be less than $15 \operatorname{deg} \mathrm{C}$ at the surface of the amorphous and ferrite cores, indicating that magnetic saturation in the core material will not be a problem.

\section{ACKNOWLEDGEMENT}

The authors are grateful to Eiji Ezura and Shigeshi Ninomiya of KEK for the valuable advice they provided in carrying out this project.

\section{REFERENCES}

[1] K. Muto et al., "Ferrite Loaded Untuned RF Cavity for Synchrotron", Proc. of the 1989 Particle Accelerator Conference.

[2] T. Nagayama, "A Prototype Non-Resonant RF Cavity for a Medical Synchrotron”, Proc. of Multi-GeV High-Performance Accelerators and Related Technology, p.230, Osaka, March 1997.

[3] S. Ninomiya, "Conceptual Design of a Non-Resonant Accelerating System for Low-Energy Proton and Heavy-Ion Accelerators", KEK Report, 92-2, April 1999 